\section{Interações medicamentosas potenciais entre idosos em uso dos anti-hipertensivos da Relação Nacional de Medicamentos Essenciais do Ministério da Saúde do Brasil}

\author{
Potential drug-drug interactions among elderly \\ using antihypertensives from the Brazilian List of \\ Essential Medicines
}

Pablo Mibielli 1

Suely Rozenfeld 1

Guacira Corrêa de Matos 2

Francisco de Assis Acurcio 3

\author{
${ }^{1}$ Escola Nacional de Saúde \\ Pública Sergio Arouca \\ Fundação Oswaldo Cruz, \\ Rio de Janeiro, Brasil. \\ 2 Faculdade de Farmácia, \\ Universidade Federal do Rio \\ de Janeiro, Rio de Janeiro, \\ Brasil. \\ 3 Faculdade de Farmácia, \\ Universidade Federal de \\ Minas Gerais, Belo Horizonte, \\ Brasil. \\ Correspondência \\ S. Rozenfeld \\ Escola Nacional de Saúde \\ Pública Sergio Arouca, \\ Fundação Oswaldo Cruz. \\ Rua Leopoldo Bulhões 1480, \\ Rio de Janeiro, RJ 21041-210, \\ Brasil. \\ rozenfel@ensp.fiocruz.br
}

\begin{abstract}
The aim of this study was to estimate the prevalence of potential interactions between antihypertensives and other drugs. A household survey was conducted with individuals 60 years or older residing in Rio de Janeiro, Brazil. Potential moderately or very severe drug-drug interactions with antihypertensives, documented as suspected, probable or established, were identified. A total of 577 elderly were interviewed (mean age $=72$ years), $45.2 \%$ of whom were using antihypertensives, of which $31.0 \%$ were subject to potential drug-drug interactions. Most of the interactions were moderately severe. Compared to the other elderly, those with potential drug-drug interactions showed more than fourfold odds of using five or more medicines and more than twofold odds of having been hospitalized in the previous year. Among the most frequent pairs of interactions, $75 \%$ cause a reduction in the hypotensive effect (65/87), which can result in low effectiveness of blood pressure control, prescribing of more drugs, and risk of other adverse events and interactions.
\end{abstract}

Drug Interactions; Antihypertensive Agents;

Drugs; Aged

\section{Resumo}

O objetivo deste estudo foi estimar a prevalência de interações medicamentosas potenciais entre anti-hipertensivos e outros fármacos. Foi realizado um inquérito domiciliar com pessoas de 60 anos ou mais de idade, residentes no Rio de Janeiro, Brasil. Foram identificadas as interações medicamentosas potenciais entre os antihipertensivos com evidência estabelecida, provável ou suspeita e com gravidade moderada ou elevada. Foram entrevistados 577 idosos (média de idade = 72 anos), 45,2\% dos quais em uso de anti-hipertensivos, sendo 31,0\% deles sujeitos a interações medicamentosas potenciais. A maioria das interações foi moderadamente grave. Comparados aos demais, os sujeitos às interações medicamentosas potenciais têm chance acima de 4 vezes de usar 5 ou mais medicamentos e acima de duas vezes de ter sido hospitalizado no ano anterior. Entre os pares de interações mais frequentes, $75 \%$ produzem redução do efeito hipotensivo (65/87), o que pode resultar em baixa efetividade no controle da pressão arterial, prescrição de mais medicamentos e risco de outros efeitos adversos e de interações.

Interações de Medicamentos; Anti-Hipertensivos; Medicamentos; Idoso 


\section{Introdução}

O tratamento das enfermidades deve ser feito preferencialmente com o menor número de medicamentos. Porém, em muitos casos e por diversos motivos, é necessário o uso concomitante de dois ou mais medicamentos, o que traz a possibilidade de interação entre eles. Dos eventos adversos que podem comprometer a qualidade da assistência à saúde, as interações medicamentosas ocupam lugar de destaque e têm sido pouco estudadas no Brasil.

A maioria dos estudos sobre o tema investiga o potencial para interações, possivelmente pela dificuldade de serem obtidas evidências da sua real ocorrência. Por outro lado, o estudo das interações medicamentosas potenciais (IMP), isto é, passíveis de ocorrência, possibilita uma análise do risco ao qual a população estudada está exposta.

Um subgrupo populacional com risco elevado de sofrer IMP é formado pelos pacientes idosos que, com frequência, utilizam muitos medicamentos, apresentam comorbidades, redução da função hepática e renal, além de dificuldades para manter o status nutricional adequado ${ }^{1}$.

A hipertensão arterial sistêmica (HAS) é doença crônica não transmissível de prevalência elevada. Analisando os dados de 13 estudos de base populacional realizados em diferentes cidades brasileiras a partir de 1990, a prevalência na população geral ficou entre 19,8\% e 44,4\%, conforme a cidade estudada. Contudo, na faixa etária com 60 anos ou mais, a proporção dos que referiram ter diagnóstico clínico de hipertensão, entre os que tiveram pressão arterial aferida nos últimos dois anos, situou-se entre $39,0 \%$ e $59,0 \% 2$.

Segundo as Diretrizes Brasileiras de Hipertensão Arterial, estão disponíveis no Brasil seis classes de fármacos anti-hipertensivos, envolvendo mais de dez mecanismos de ação diferentes 3 . Dependendo do estágio de gravidade da doença, a monoterapia não é suficiente, e pode ser necessário associar até três medicamentos, o que eleva o risco para ocorrência de IMP.

Assim, o estudo teve como objetivo estimar a prevalência de interações medicamentosas potenciais entre os fármacos apresentados como anti-hipertensivos na Relação Nacional de Medicamentos Essenciais (RENAME 2010) 4, e entre eles e quaisquer outras substâncias ativas.

$\mathrm{O}$ estudo foi feito com base nos medicamentos usados por amostra de pessoas com 60 anos de idade ou mais, residentes no Rio de Janeiro. Pretendeu-se também identificar os fatores associados à ocorrência de IMP.

\section{Métodos}

\section{Fonte dos dados e população de estudo}

Os dados analisados provêm de um inquérito realizado em 2003 cujo objetivo primeiro foi descrever o perfil de medicamentos usados por aposentados e pensionistas vinculados ao Regime Geral de Previdência Social do Instituto Nacional do Seguro Social (INSS), Ministério da Previdência e Assistência Social (MPAS), com idade igual ou superior a 60 anos ${ }^{5}$. As informações foram coletadas junto aos indivíduos selecionados por meio de entrevistas domiciliares. Aqui, serão analisados os dados de residentes na cidade do Rio de Janeiro.

Valendo-se dos 456.628 aposentados e pensionistas residentes no Rio de Janeiro, extraiu-se amostra de 577 indivíduos. A seleção foi efetuada pelo uso da Empresa de Tecnologia e Informações da Previdência Social (DATAPREV), com base no cadastro de beneficiários, por amostra aleatória simples, considerando-se nível de confiança de $95 \%$ e taxas de resposta de $75 \%$. Inicialmente, foram sorteados 800 indivíduos. Para substituir perdas inevitáveis, por falecimento ou mudança para endereços não localizados, a amostra original foi acrescida de 100 indivíduos. A taxa de resposta foi $71 \% 5$.

São apresentadas as análises correspondentes a uma subamostra constituída pelos 261 $(45,2 \%)$ entrevistados usuários de anti-hipertensivos. Tais indivíduos foram considerados potenciais sujeitos a interações farmacológicas, desde que usassem, no mínimo, um segundo fármaco, e que o mesmo apresentasse interação descrita na literatura com o anti-hipertensivo declarado como usado por cada entrevistado. As definições operacionais de anti-hipertensivo e de interação farmacológica empregadas na análise dos dados estão descritas no próximo item.

Os questionários para entrevista domiciliar contavam com três blocos de perguntas sobre: características sociais e demográficas dos entrevistados; indicadores das condições de saúde e uso de serviços de saúde; e uso de medicamentos num período recordatório de 15 dias. A coleta de dados foi feita por graduandos em farmácia, treinados segundo um manual de instrução preparado para o estudo.

O inquérito foi aprovado pelo Comitê de Ética em Pesquisa da Fundação Oswaldo Cruz (parecer no 79/02), com garantia de sigilo e confidencialidade das informações e obtenção de termo de consentimento livre e esclarecido assinado pelos participantes. 
Definição de interação medicamentosa potencial e fontes de informação para seleção dos pares de fármacos

IMP pode ser definida como a possibilidade de um medicamento alterar a intensidade do efeito farmacológico de outro medicamento administrado concomitantemente 6 .

A seguir, são apresentadas as fontes bibliográficas nas quais foram definidos os anti-hipertensivos e os fármacos com os quais eles podem interagir.

- Anti-hipertensivos: trata-se de fármacos com ação no sistema cardiovascular empregados no tratamento da hipertensão. Os anti-hipertensivos foram selecionados com base na RENAME 4 . Ela é um importante instrumento para a assistência farmacêutica, construída com base nas necessidades nacionais, e engloba fármacos com comprovadas segurança e eficácia terapêutica. Embora o mercado farmacêutico tenha outras substâncias comercializadas com indicação para tratamento da hipertensão arterial, a escolha da RENAME como fonte de informação objetivou fixar, como base para o estudo, o cenário mais favorável, no qual são considerados os fármacos mais adequados ao controle da doença. A opção significou assumir alguma subestimação na prevalência de possíveis interações entre os participantes. Os anti-hipertensivos selecionados pertencem a seis classes diferentes, e são os seguintes: espironolactona e hidroclorotiazida (diuréticos); atenolol, metildopa, metoprolol e propranolol (bloqueadores adrenérgicos); anlodipino e verapamil (bloqueadores de canais de cálcio); hidralazina e nitroprusseto de sódio (vasodilatadores diretos); captopril e enalapril (inibidores da enzima conversora de angiotensina) e losartana potássica (antagonista de receptores de angiotensina).

- Fármacos que interagem com os anti-hipertensivos: a fonte de informação foi o Drug Interaction Facts 7 que classifica as interações em cinco níveis de evidência (estabelecida, provável, suspeita, possível e improvável) e três de gravidade (leve, moderada e elevada). Para esse estudo foram selecionadas apenas as interações classificadas em nível de evidência estabelecida, provável ou suspeita, e de gravidade moderada ou elevada.

\section{Análise de dados}

Criaram-se dois bancos de dados, o de medicamentos, onde cada linha tem informações sobre um fármaco, ocupando cada participante tantas linhas quantos forem os fármacos que ele utiliza; e o de informações sociais e de saúde, onde cada linha corresponde a um participante. Para relacionar as informações neles contidas, o banco de medicamentos foi horizontalizado, e ambos foram agregados. Após, foram criadas colunas com as variáveis correspondentes a cada par fármacofármaco (categorias: presente/ausente). O procedimento permitiu classificar cada participante quanto à presença ou ausência de, no mínimo, uma IMP. Assim foi criada a variável dependente. As definições de anti-hipertensivo e de interações foram as descritas acima. A lista dos fármacos e das interações e o script para construção da variável podem ser obtidos mediante solicitação aos autores. As variáveis independentes analisadas foram: sexo, idade, escolaridade, número de pessoas na residência, número de medicamentos usados, internação hospitalar no último ano, número de consultas médicas nos últimos 12 meses, estado de saúde autorreferido, participação em plano de saúde, ter estado acamado nos últimos 15 dias.

Para identificar possíveis fatores associados às IMP, foi realizada análise logística univariada, e calculados odds ratio (OR) com respectivos intervalos de 95\% de confiança (IC95\%). Os dados são apresentados como frequências absolutas e relativas. $\mathrm{Na}$ análise multivariada por regressão logística, incluíram-se as variáveis que, na etapa anterior, apresentaram diferenças entre os subgrupos com $\mathrm{p}<0,20$. Na modelagem, variáveis correlacionadas entre si foram excluídas do modelo. Os OR e respectivos IC95\% foram calculados. Em todos os procedimentos, foram consideradas significantes as diferenças entre os subgrupos de $\mathrm{p}<0,05$. Os dados foram organizados e analisados no SPSS, versão 17.0 (SPSS Inc., Chicago, Estados Unidos).

\section{Resultados}

Na amostra, composta por 577 indivíduos, houve predominância do sexo feminino (61\%). A média de idade foi 72 anos, variando de 60 a 94 anos. A maioria dos participantes $(64,1 \%)$ referiu ter doenças diretamente relacionadas ao sistema cardiovascular. O número de fármacos usados variou de 0 a 24 (mediana $=3$ ). Do total de entrevistados, $261(45,2 \%)$ eram usuários de antihipertensivos e, entre eles, 81 (31,0\%) estavam sujeitos a IMP, sendo $74,1 \%$ a uma interação, $17,3 \%$ a duas e $8,6 \%$ a três ou mais.

A Tabela 1 caracteriza os participantes sujeitos à IMP, segundo variáveis demográficas, sociais e de saúde. A proporção de indivíduos sujeitos à IMP foi maior nos seguintes subgrupos: os usuários de 5 ou mais medicamentos; os hospitalizados no ano anterior; os submetidos a 6 ou mais 
Caracterização dos participantes sujeitos a interações medicamentosas potenciais (IMP), segundo variáveis demográficas, sociais e de saúde, entre aposentados, usuários de anti-hipertensivos, com 60 anos de idade ou mais. Município do Rio de Janeiro, Brasil, 2003 ( $N=261)$.

\begin{tabular}{|c|c|c|}
\hline Características & Com IMP (\%) & OR (IC95\%) \\
\hline \multicolumn{3}{|l|}{ Sexo } \\
\hline Masculino & $30(32,3)$ & $0,92(0,53-1,58)$ \\
\hline Feminino & $51(30,4)$ & \\
\hline \multicolumn{3}{|l|}{ Idade (anos) } \\
\hline $60-74$ & $51(31,5)$ & $0,95(0,55-1,63)$ \\
\hline$\geq 75$ & $30(30,3)$ & \\
\hline \multicolumn{3}{|l|}{ Escolaridade } \\
\hline Até 1ㅇ Grau incompleto & $55(31,3)$ & $1,03(0,59-1,81)$ \\
\hline 1ㅇ Grau completo e mais & $26(30,6)$ & \\
\hline \multicolumn{3}{|l|}{ Mora só } \\
\hline Sim & $11(26,2)$ & $0,75(0,36-1,58)$ \\
\hline Não & $70(32,1)$ & \\
\hline \multicolumn{3}{|c|}{ Número de medicamentos utilizados * } \\
\hline 5 ou mais & $59(46,8)$ & $4,52(2,54-8,04)$ \\
\hline $1-4$ & $22(16,3)$ & \\
\hline \multicolumn{3}{|c|}{ Internação hospitalar no último ano * } \\
\hline Sim & $25(50,0)$ & $2,82(1,50-5,31)$ \\
\hline Não & $55(26,2)$ & \\
\hline \multicolumn{3}{|c|}{ Consultas médicas nos últimos 12 meses ** } \\
\hline 6 ou mais & $49(39,2)$ & $2,08(1,22-3,54)$ \\
\hline $1-5$ & $32(23,7)$ & \\
\hline \multicolumn{3}{|c|}{ Estado de saúde autorreferido *** } \\
\hline Muito ruim ou ruim & $16(45,7)$ & $2,86(1,25-6,57) \#$ \\
\hline Regular & $40(31,7)$ & \\
\hline Muito bom ou bom & $20(22,7)$ & \\
\hline \multicolumn{3}{|l|}{ Possui plano de saúde } \\
\hline $\operatorname{Sim}$ & $29(28,4)$ & $1,20(0,70-2,07)$ \\
\hline Não & $51(32,3)$ & \\
\hline \multicolumn{3}{|c|}{ Esteve de cama nos últimos 15 dias * } \\
\hline $\operatorname{Sim}$ & $18(58,1)$ & $3,67(1,70-7,93)$ \\
\hline Não & $63(27,4)$ & \\
\hline
\end{tabular}

IC95\%: intervalo de 95\% de confiança; OR: odds ratio.

${ }^{\star} \mathrm{p} \leq 0,001$;

$\star * \mathrm{p}=0,007$

$\star * * p=0,040$;

\# IC95\% para a categoria muito ruim ou ruim em relação à categoria muito bom ou bom.

consultas médicas nos últimos 12 meses; os que consideravam seu estado de saúde muito ruim ou ruim; e os acamados nos últimos 15 dias. As diferenças foram estatisticamente significativas.

Foram observados 26 pares diferentes de IMP entre os usuários de anti-hipertensivos, totalizando 110 interações em 81 indivíduos, sendo 23 de gravidade elevada (20,9\% das interações) e 87 de gravidade moderada $(79,1 \%$ das interações).
A Tabela 2 apresenta os pares de IMP mais encontrados, e a frequência de cada par no total de usuários de anti-hipertensivos $(\mathrm{n}=261$ ). Observa-se que o número total de pares encontrados na amostra supera o número de sujeitos expostos à IMP, pois vários deles estão expostos a duas ou mais interações. As classes de fármacos anti-hipertensivos mais encontradas nas interações foram os inibidores da enzima conversora 
Interações medicamentosas potenciais (IMP) entre aposentados, usuários de anti-hipertensivos, com 60 anos de idade ou mais. Município do Rio de Janeiro, Brasil, $2003(\mathrm{~N}=261)$

\begin{tabular}{|c|c|c|}
\hline Pares de fármacos * & Consequências clínicas ** & $\begin{array}{l}\text { Frequência de IMP } \\
\text { na amostra (\%) }\end{array}$ \\
\hline Maleato de enalapril e ácido acetilsalicílico & Redução do efeito hipotensivo e vasodilatador & $25(9,6)$ \\
\hline Captopril e ácido acetilsalicílico & Redução do efeito hipotensivo e vasodilatador & $17(6,5)$ \\
\hline Atenolol e ácido acetilsalicílico & $\begin{array}{l}\text { Efeitos anti-hipertensivos dos betabloqueadores } \\
\text { podem ser atenuados pelos salicilatos }\end{array}$ & $17(6,5)$ \\
\hline Maleato de enalapril e cloridrato de amilorida & Elevação da concentração sérica de $K+$ em pacientes de risco & $6(2,3)$ \\
\hline Cloridrato de propranolol e ácido acetilsalicílico & $\begin{array}{l}\text { Efeitos anti-hipertensivos dos betabloqueadores } \\
\text { podem ser atenuados pelos salicilatos }\end{array}$ & $6(2,3)$ \\
\hline Espironolactona e captopril & Elevação da concentração sérica de $\mathrm{K}+$ em pacientes de risco & $5(1,9)$ \\
\hline Espironolactona e digoxina & $\begin{array}{l}\text { Diurético pode atenuar o efeito inotrópico positivo da digoxina. } \\
\text { Digoxina pode ser aumentada no soro }\end{array}$ & $4(1,5)$ \\
\hline Hidroclorotiazida e digoxina & $\begin{array}{c}\text { Distúrbios eletrolíticos induzidos pela tiazida podem } \\
\text { predispor a arritmias induzidas pelos digitálicos }\end{array}$ & $4(1,5)$ \\
\hline Hidroclorotiazida e furosemida & $\begin{array}{c}\text { Ambos os grupos têm efeitos sinérgicos que podem resultar } \\
\text { em diurese intensa e anormalidades eletrolíticas graves }\end{array}$ & $3(1,1)$ \\
\hline
\end{tabular}

* Consideradas as identificadas em, no mínimo, três participantes;

** Fonte: Tatro 7 .

de angiotensina, os bloqueadores adrenérgicos e os diuréticos. Um dos pares de IMP identificados envolveu dois agentes anti-hipertensivos, espironolactona e captopril. Os pares mais encontrados envolveram o ácido acetilsalicílico (AAS). Eram compostos com AAS+enalapril, AAS+captopril ou AAS+atenolol, tendo sido registrados 25, 17 e 17 vezes, respectivamente.

Cerca de $10 \%$ dos usuários de anti-hipertensivos (25 indivíduos) relatou uso simultâneo de maleato de enalapril e ácido acetilsalicílico, que pode levar à redução do efeito hipotensivo e vasodilatador do enalapril. A mesma consequência clínica pode ocorrer com diversos outros pares de fármacos. Entre os pares mais frequentes $75 \%$ (65/87) produzem redução do efeito anti-hipertensivo (Tabela 2).

Conforme apresentado na Tabela 3, os indivíduos com interações medicamentosas potenciais têm chance acima de $300 \%$ de ter usado 5 ou mais medicamentos em relação aos usuários de até 4 medicamentos (OR = 4,38; IC95\%: 2,34$8,21)$, independente de ele ter sido internado no ano anterior ou de referir pior estado de saúde. O sexo e a idade não alteraram significativamente as estimativas.

\section{Discussão}

Na amostra composta por 577 indivíduos acima de 60 anos de idade, beneficiários da Previdência Social residentes no Rio de Janeiro, 45,2\% eram usuários de anti-hipertensivos. Nesse grupo formado por 261 indivíduos, 31,0\% usavam substâncias que, com elevado grau de evidência científica, interagem com os anti-hipertensivos, podendo causar eventos adversos moderados ou graves. Os inibidores da enzima conversora de angiotensina, os bloqueadores adrenérgicos e os diuréticos foram as principais classes de agentes anti-hipertensivos envolvidos nas IMP. A restrição do estudo apenas aos entrevistados em uso de anti-hipertensivos listados na RENAME como os mais eficazes e seguros teve o intuito de explorar o cenário mais otimista. Possivelmente, caso a análise abordasse o conjunto dos fármacos usados pelos participantes, a estimativa de ocorrência de IMP ultrapassaria os $50 \% 8$.

Os indivíduos que fizeram uso de 5 ou mais medicamentos estiveram internados no último ano, consultaram-se com médicos 6 ou mais vezes no último ano, referiram pior estado de saúde e estiveram acamados nos 15 dias antes das entrevistas, apresentaram maior proporção de IMP. Tal achado sugere a existência de subgrupos mais vulneráveis entre os idosos. Neles, a terapia farmacológica deve se redobrar em cuidados. 
Fatores associados às interações medicamentosas potenciais (IMP) entre aposentados, usuários de anti-hipertensivos, com 60 anos de idade ou mais. Município do Rio de Janeiro, Brasil, 2003 ( $N=261$ ).

\begin{tabular}{lcc}
\hline Variáveis & OR brutas (IC95\%) & OR ajustadas (IC95\%) \\
\hline $\begin{array}{l}\text { Número de medicamentos utilizados } \\
1-4\end{array}$ & 1,00 & 1,00 \\
5 ou mais & $4,52(2,54-8,04)$ & $4,38(2,34-8,21)$ \\
Internação hospitalar no último ano & 1,00 & 1,00 \\
Não & $2,82(1,50-5,31)$ & $2,42(1,20-4,91)$ \\
Sim & 1,00 & 1,00 \\
Estado de saúde autorreferido & $1,58(0,85-2,95)$ & $0,98(0,49-1,95)$ \\
Muito bom ou bom & $2,86(1,25-6,57)$ & $1,71(0,69-4,26)$ \\
Regular & & \\
Muito ruim ou ruim & & \\
\hline
\end{tabular}

IC95\%: intervalo de 95\% de confiança; OR: odds ratio.

Há maior proporção com IMP entre os hospitalizados no ano anterior à entrevista $(50 \% v s$. $26 \%$ ) ou os que se consultaram mais vezes com médicos (40\% vs. 24\%), o que faz supor estado de saúde mais precário. Em indivíduos com múltiplas doenças pode haver necessidade de mais fármacos, não sendo possível evitar a ocorrência de IMP. Ainda assim, o conhecimento a respeito delas é importante para controlar e prevenir os possíveis efeitos adversos, entre eles, a redução do efeito hipotensor. Ele ocorre, entre outras situações, com o uso concomitante de inibidores da enzima conversora de angiotensina e de AAS e pode ser contornado com: diminuição da dose de AAS para menos de 100mg por dia; substituição do inibidor da enzima conversora de angiotensina por um antagonista dos receptores de angiotensina, ou substituição do AAS por outro agente antiagregante plaquetário 7,9.

O número de medicamentos em uso é preditor independente de IMP, achado que converge com outros estudos 8,10,11, mesmo aqueles que incluem população de adultos jovens 12 . É esperado que o aumento do número de fármacos favoreça o surgimento de interações entre eles. Ainda assim, revelar a associação e dimensionar o aumento do risco - aqui, acima de $300 \%$ - é relevante porque chama a atenção para a necessidade de prescrições mais parcimoniosas.

A relação entre o aumento da idade e a ocorrência de IMP apresenta aspectos que merecem mais investigação. Assim como Secoli et al. ${ }^{8}$, não se encontrou diferença significativa na probabilidade de apresentar IMP entre o grupo com 60 a 74 anos e o grupo com 75 anos ou mais de idade. Já outros autores 13 observaram maior frequên- cia das IMP entre as mulheres de até 79 anos de idade, em comparação com os grupos com 80 anos e mais de idade. É possível que o cuidado na prescrição entre os muito idosos reduza o número de fármacos prescritos e com isso o risco de sofrer IMP.

Uma das dificuldades que envolvem o estudo das interações entre fármacos está na definição da fonte de informação, seja ela compilação de textos, revisões sistemáticas, base eletrônicas de dados, ou artigos isolados. As estimativas de frequência de IMP podem variar na dependência da fonte de informação empregada para identificar as interações a serem analisadas, suas consequências e sua gravidade. Mesmo empregando a mesma fonte, pode haver diferenças nos critérios escolhidos. Das cinco categorias de relevância clínica da interação, oferecidas pela base de dados empregada por nós 7 , foram consideradas as de gravidades moderada ou severa. A consideração apenas das interações com maior relevância clínica leva a estimativas de prevalências substancialmente menores 14 .

Ao comparar estudos realizados com fontes diferentes de informação, a preocupação com os critérios de escolha das IMP deve ser ainda maior, visto que não se conhece o grau de concordância entre elas. Vitry 15 selecionou 50 fármacos e listou as interações entre eles segundo quatro fontes de informação diferentes. Das interações classificadas com nível de significância estabelecida ou provável no Drug Interaction Facts, 14,6\% não estavam incluídas no Micromedex, e entre as interações comuns a duas fontes de informação, 33\% das classificadas como de gravidade elevada no Micromedex foram classi- 
ficadas como de gravidade moderada no Drug Interaction Facts.

Entre as fontes de informação disponíveis sobre interações, a escolha recaiu sobre o Drug Interaction Facts 7, por ser fonte sensível e específica, citada repetidamente em artigos da área; por oferecer literatura científica para embasar as evidências sobre cada um dos fármacos; por estruturar as interações segundo a gravidade e o grau de evidência. O Drug Interaction Facts é parte da série de publicações Facts \& Comparisons, e ambos têm painéis revisores formados por profissionais médicos e farmacêuticos, vários deles com vínculos em universidades norte-americanas. Além disso, disponibiliza atualizações online e a consulta é facilitada pelo formato.

Os inibidores da enzima conversora de angiotensina estão presentes em três dos quatro pares de IMP mais frequentes no presente estudo. Em outras avaliações de população idosa 8,11,16, essa classe de medicamentos também aparece no topo das listas de IMP mais frequentes. No entanto, em amostra que inclui adultos jovens 14,17 , a digoxina e os diuréticos figuram entre as interações mais frequentes, deixando os inibidores da enzima conversora de angiotensina em posições inferiores. Ao comparar os inibidores da enzima conversora da angiotensina com os bloqueadores de receptores de angiotensina parece não haver diferenças significativas entre eles, quanto ao controle da pressão arterial, às taxas de mortalidade e à ocorrência de eventos cardiovasculares mais graves 18 . Portanto, os bloqueadores de receptores de angiotensina podem ser considerados como alternativa.

O AAS, amplamente utilizado como antiagregante plaquetário, e que inibe a ocorrência de eventos cardiovasculares, apareceu nesse estudo envolvido em quatro dos cinco pares de IMP mais frequentes. A interação entre AAS e inibidores da enzima conversora de angiotensina tem sido identificada como relevante em outros estudos em população idosa 8,10. A interação é plausível 9 , pois os inibidores da enzima conversora de angiotensina provocam aumento da produção de prostaglandinas, que têm ação vasodilatadora que pode ser bloqueada pelo AAS, cuja inibição da enzima ciclooxigenase e bloqueio da produção de prostaglandinas conta com evidências consistentes. A ocorrência de interações, conforme as diferentes doses diárias usadas de AAS, deve ser levada em conta, embora haja indícios de que, por enquanto, esse é um assunto controverso ${ }^{7}$.

É importante notar a frequência de IMP antagônica, no sentido de diminuição do efeito dos anti-hipertensivos. Entre os pares de fármacos mais frequentes, com ação sinérgica ou antagô- nica, 75\% produzem redução do efeito hipotensivo (65/87), o que significa quatro entre as nove IMP mais frequentes. Isso pode se traduzir por baixa efetividade do controle da pressão arterial, acréscimos de outros anti-hipertensivos, risco de novos efeitos adversos e outras interações. Cerca de $10 \%$ da amostra usa maleato de enalapril e AAS, que foi a combinação de fármacos mais encontrada, cujo resultado é a redução do efeito hipotensivo e vasodilatador.

No campo complexo do estudo das interações é preciso estar atento à possível ocorrência de efeitos não desejados, decorrentes não só das interações entre medicamentos, como também das interações entre medicamento e doença, e do uso inapropriado para grupos de risco. Entre os fármacos utilizados no sistema cardiovascular, segundo o critério STOPP (Screening Tool of Older Persons' potentially inappropriate Prescription), o uso de betabloqueadores é inapropriado em pacientes com doença pulmonar obstrutiva crônica, assim como o AAS é inapropriado aos pacientes sem história de sintomas coronarianos, cerebrais ou vasculares periféricos ou de eventos oclusivos ${ }^{19}$. Logo, identificado o problema, é fundamental adotar medidas adequadas, corrigindo dosagens ou examinando alternativas terapêuticas.

O aprimoramento da terapia com fármacos entre idosos se beneficiaria com o desenvolvimento de listas baseadas em evidências científicas para orientar objetivamente as práticas de prescrição e o monitoramento não apenas com relação às IMP, mas também quanto aos medicamentos inapropriados para idosos. Há ferramentas de orientação em alguns países, entretanto é necessário o desenvolvimento de listas nacionais, construídas com base em fármacos comercializados em cada país 20.

Um dos aspectos positivos do estudo é ter focado tema pouco tratado entre nós e de difícil abordagem. Entre as dificuldades, destaca-se o fato de que nem todas as combinações entre os múltiplos fármacos existentes, ou comumente usados de modo simultâneo, têm estudos para sustentar evidências científicas sobre a ocorrência, ou não, de IMP. Outra dificuldade está na ausência de informação válida sobre as diferentes posologias empregadas, mesmo em estudos epidemiológicos que abordam o uso de medicamentos. Para ilustrar, o uso de AAS em comprimidos de 350mg parece atenuar o efeito vasodilatador do enalapril e outros efeitos dependentes de prostaglandinas por mais de 24 horas 21 .

Investigamos o uso de medicamentos entre os aposentados pela Previdência Social, o que não exclui extrapolar os dados para outras populações, com os devidos cuidados. Com relação 
aos residentes na cidade do Rio de Janeiro, os beneficiários do sistema de aposentadoria oficial com idade igual ou superior a 60 anos correspondiam a $64 \%$ de habitantes da cidade na mesma faixa etária, no ano do estudo. Com isso, espera-se estimativa semelhante de prevalência de IMP para o conjunto da população idosa da cidade.

Entre as limitações, ressalta-se o fato de o estudo basear-se em informação obtida em entrevistas com os participantes, e não em registros dos tratamentos nos prontuários médicos. Vale dizer, não é possível avaliar a relevância clínica dos eventos que, de fato, ocorreram. Além disso, não se pode garantir que os medicamentos envolvidos em IMP foram efetivamente utilizados de modo concomitante, dentro do período recordatório de 15 dias. Para atenuar possíveis distorções decorrentes do fato de que nem todos os medicamentos autorreferidos teriam sido empregados, foi solicitado aos entrevistados comprovação de uso do medicamento, com apresentação de receita médica, bula ou embalagem. Possível viés de lembrança pode ter ocorrido, em particular por se tratar de amostra de idosos, e resultado em sub-registro de produtos e subestimação da prevalência de IMP. A comparação com estimativas de outros estudos não deve ter sido prejudicada, uma vez que os procedimentos adotados e o período recordatório costumam ser semelhantes. Embora a coleta de dados seja de 2003, os fármacos estudados, tirante a losartana, já eram amplamente empregados.

Em resumo, a prevalência de indivíduos utilizando substâncias que interagem com anti-hi- pertensivos entre os idosos foi elevada (31,0\%). A chance de usar 5 ou mais medicamentos e a de ter sido hospitalizado no ano anterior ao estudo foi cerca de 4 vezes maior, e cerca de duas vezes maior, respectivamente, entre os que apresentaram interação medicamentosa potencial quando comparados aos demais entrevistados. As interações medicamentosas podem ser consideradas um tipo de reação adversa aos medicamentos e são intercorrências que devem ser conhecidas, monitoradas e controladas.

Os resultados encontrados sugerem que se deva restringir o número de fármacos prescritos. Além de estar atento para identificar uma possível falta de efetividade do tratamento anti-hipertensivo provocada por interação entre fármacos. Tais medidas se somariam aos esforços em curso do Governo Federal e à política de controle da hipertensão arterial. O combate ao sedentarismo e o aumento do acesso a medicamentos gratuitos na rede de farmácias se dirigem ao controle dos fatores de risco e ao aprimoramento do acesso ao tratamento farmacológico 22 . A prescrição adequada e o monitoramento de possíveis interações medicamentosas se voltam para melhor controle dos níveis pressóricos e redução das hospitalizações decorrentes das complicações da doença.

No plano da investigação, é importante que outros estudos sobre interações medicamentosas surjam em nosso meio, objetivando ampliar o conhecimento sobre o problema, não apenas com o uso dos anti-hipertensivos, mas também de outras classes terapêuticas usadas no controle de doenças crônicas não transmissíveis. 


\section{Resumen}

El objetivo de este estudio fue estimar la prevalencia de interacciones medicamentosas potenciales entre antihipertensivos y otros fármacos. Se realizó una encuesta domiciliaria con personas de 60 años o edad más avanzada, residentes en Río de Janeiro, Brasil. Se identificaron interacciones medicamentosas potenciales entre antihipertensivos con una evidencia establecida, probable o sospechosa y con una gravedad moderada o elevada. Fueron entrevistadas 577 personas de la tercera edad (media de edad = 72 años), un 45,2\% de los cuales tomaba antihipertensivos, estando un 31,0\% de ellos sujetos a interacciones medicamentosas potenciales. La mayoría de las interacciones fue moderadamente grave. Comparados con los demás, los sujetos potenciales a interacciones con medicamentos tienen una probabilidad 4 veces superior de usar 5 o más medicamentos $y$ dos veces mayor de haber sido hospitalizado durante el año anterior. Los hospitalizados en el último año tienen una probabilidad 2 veces superior de exponerse a interacciones medicamentosas potenciales. Entre los pares de interacciones más frecuentes, un 75\% producen reducción del efecto hipotensivo (65/87), lo que puede resultar en una baja efectividad en el control de la presión arterial, prescripción de más medicamentos y riesgo de otros efectos adversos y de interacciones.

Interacciones de Drogas; Antihipertensivos; Drogas; Anciano

\section{Referências}

1. Mallet L, Spinewine A, Huang A. The challenge of managing drug interactions in elderly people. Lancet 2007; 370:185-91.

2. Passos VMA, Assis TD, Barreto SM. Hipertensão arterial no Brasil: estimativa de prevalência a partir de estudos de base populacional. Epidemiol Serv Saúde 2006; 15:35-45.

3. Sociedade Brasileira de Cardiologia. VI diretrizes brasileiras de hipertensão. Rev Bras Hipertens 2010; 17:7-10.

4. Departamento de Assistência Farmacêutica e Insumos Estratégicos, Secretaria de Ciência, Tecnologia e Insumos Estratégicos, Ministério da Saúde. Relação Nacional de Medicamentos Essenciais: RENAME. 7ạ Ed. Brasília: Ministério da Saúde; 2010. (Série B. Textos Básicos de Saúde).

5. Acurcio FA, Rozenfeld S, Ribeiro AQ, Klein CH, Moura CS, Andrade CR. Utilização de medicamentos por aposentados brasileiros. 1 - Metodologia e resultados de cobertura de inquérito multicêntrico. Cad Saúde Pública 2006; 22:87-96.

\section{Colaboradores}

P. Mibielli, S. Rozenfeld, G. C. Matos e F. A. Acurcio contribuíram para a concepção do estudo, a interpretação dos resultados, a revisão crítica do texto, aprovação da versão final a ser publicada. P. Mibielli e S. Rozenfeld realizaram a análise dos dados e a redação do artigo.

\section{Agradecimentos}

Os autores agradecem ao Programa de Pós-graduação em Epidemiologia em Saúde Pública, da Escola Nacional de Saúde Pública Sergio Arouca/Fiocruz. O estudo é parte da Dissertação de Mestrado do autor P. Mibielli.
6. Nies AS. Principles of therapeutics. In: Hardman JG, Gilman AG, Limbird L, editors. Goodman \& Gilman's the pharmacological basis of therapeutics. International edition. New York: McGraw-Hill Professional; 1996. p. 43-63.

7. Tatro DS. Drug interaction facts. St. Louis: Wolters Kluwer Health; 2011.

8. Secoli SR, Figueras A, Lebrão ML, Lima FD, Santos JLF. Risk of potential drug-drug interactions among Brazilian elderly. Drugs Aging 2010; 27:759-70.

9. Cleland JG, John J, Houghton T. Does aspirin attenuate the effect of angiotensin-converting enzyme inhibitors in hypertension or heart failure? Curr Opin Nephrol Hypertens 2001; 10:625-31.

10. Doubova SV, Reyes-Morales H, Torres-Arreola LP, Suárez-Ortega M. Potential drug-drug and drugdisease interactions in prescriptions for ambulatory patients over 50 years of age in family medicine clinics in Mexico City. BMC Health Serv Res 2007; $7: 147$. 
11. Bacic-Vrca V, Marusic S, Erdeljic V, Falamic S. The incidence of potential drug-drug interactions in elderly patients with arterial hypertension. Pharm World Sci 2010; 32:815-21.

12. Carter BL, Lund BC, Hayase N, Chrischilles E. The extent of potential antihypertensive drug interactions in a Medicaid population. Am J Hypertens 2002; 15:953-7.

13. Venturini CD, Engroff P, Ely LS, Zago LFA, Schroeter G, Gomes I, et al. Gender differences, polypharmacy, and potential pharmacological interactions in the elderly. Clinics 2011; 66:1867-72.

14. Magro L, Conforti F, Del Zotti F, Leone R, Iorio ML, Meneghelli I, et al. Identification of severe potential drug-drug interactions using an Italian general-practitioner database. Eur J Clin Pharmacol 2008; 64:303-9.

15. Vitry AI. Comparative assessment of four drug interaction compendia. Br J Clin Pharmacol 2006; 63:709-14.

16. Tulner LR, Frankfort SV, Gijsen PT, Campen PCM, Koks CHW, Beijnen JH. Drug-drug interactions in a geriatric outpatient cohort. Drugs Aging 2008; 25:343-55.

17. Moura C, Acurcio F, Belo N. Drug-drug interactions associated with length of stay and cost of hospitalization. J Pharm Pharmaceut Sci 2009; 12:266-72.
18. Powers B, Greene L, Balfe LM. Updates on the treatment of essential hypertension: a summary of AHRQ's comparative effectiveness review of angiotensin-converting enzyme inhibitors, angiotensin II receptor blockers, and direct renin inhibitors. J Manag Care Pharm 2011; 17(8 Suppl):S1-14.

19. Gallagher P, O'Mahony D. STOPP (Screening Tool of Older Persons' potentially inappropriate Prescriptions): application to acutely ill elderly patients and comparison with Beers' criteria. Age Ageing 2008; 37:673-9.

20. Guaraldo L, Cano FG, Damasceno GS, Rozenfeld S Inappropriate medication use among the elderly:a systematic review of administrative databases. BMC Geriatr 2011; 11:79.

21. Hall D, Zeitler H, Rudolph W. Counteraction of the vasodilator effects of enalapril by aspirin in severe heart failure. J Am Coll Cardiol 1992; 20:1549-55.

22. Presidência da República. SUS amplia acesso ao tratamento da hipertensão arterial. http://www2. planalto.gov.br/excluir-historico-nao-sera-migra do/sus-amplia-acesso-ao-tratamento-da-hiper tensao-arterial-1 (acessado em 15/Mai/2014).

Recebido em 05/Jul/2013

Versão final reapresentada em 17/Fev/2014

Aprovado em 21/Fev/2014 